\title{
Significance of Platelet Parameters and Troponin $T$ in Patients with Acute Coronary Syndrome
}

\author{
Authors \\ Dr G. Nataraju, Dr S.A.K. Adil, Dr R.K. Divya \\ Department of Pathology, MMC \& RI, Mysore \\ Email: divya.rk0411@gmail.com
}

\begin{abstract}
Background: Coronary heart disease (CHD) is among the top five causes of morbidity worldwide, and manifests as acute coronary syndrome (ACS). Identifying risk factors for acute coronary syndrome (ACS) is important for both diagnostic and prognostic purpose. It is postulated that individuals with a large platelets are more predisposed to occluding thrombi in coronary vessels. Larger platelets are enzymatically and metabolically more active and have a higher potential thrombotic ability as compared with smaller platelets. Recently Cardiac markers are used in the diagnosis and risk stratification of patients with chest pain and suspected acute coronary syndrome (ACS). The cardiac troponins, namely TroponinT in particular, have become the cardiac marker of choice for patients with ACS.

Objectives: The aim of this study is to investigate whether there is an association of platelet indices and cardiac Troponin $T$ with acute coronary syndrome and the significance of these parameters in the diagnostic workup of ACS.

Method: A descriptive study was done in Sri Jayadeva Institute of Cardiac science and Research (SJICSR), Mysore. A total of 100 patients admitted with diagnosis of ACS during the period January-June2016 were studied These patients were subjected to investigations of complete haemogram and Troponin $T$ as routine procedure at the time of admission as they were clinically suspected as suffering from ACS.

Results: The study revealed that majority of patients were males(72\%), the major age group affected were in the age of 41-50yrs and the mean values of Platelet Indices were MPV=7.76 fl, PDW=12.2\%, Pct $=0.16 \%$ and Platelet Count $=2.53$ lacs. The mean value of Troponin $T$ with respect to gender was $0.8 \mathrm{ng} / \mathrm{ml}$ in males and $0.61 \mathrm{ng} / \mathrm{ml}$ in females.

Conclusion: Mean Platelet Volume was not found to be a risk factor for Acute Coronary syndrome in our study. But Troponin $T$ can be considered as a prognostic marker in patients with acute coronary syndrome. Keywords: Acute coronary syndrome, Platelet Parameters, Troponin T.
\end{abstract}

\section{Introduction}

Acute coronary syndrome (ACS) is becoming the leading cause of morbidity and mortality in developing countries like India too. It is unclear if certain platelet characteristics predispose some individuals with atherosclerosis to develop ACS. Larger the platelets, they are more likely to be metabolically and enzymatically active ${ }^{(1)}$ Platelets have a major role in the pathogenesis of ACS, where plaque rupture is followed by platelet activation and thrombus formation leading to coronary artery occlusion ${ }^{(2)}$ Acute coronary syndrome (ACS) encompass a spectrum of coronary artery disease from unstable angina to 
transmural myocardial infarction ${ }^{(3)}$. Atherothrombosis is the main pathophysiological mechanism of Acute coronary Syndrome ${ }^{(4)}$. When platelets are activated they become larger in size, which can be measured by both mean platelet volume (MPV) and platelet distribution width (PDW). Larger platelets are more adhesive and tend to aggregate more than smaller ones ${ }^{(2)}$ An increase in both mean platelet volume (MPV) and platelet distribution width (PDW) due to platelet activation, resulting from platelet swelling and pseudopodia formation was hypothesized. ${ }^{(5)}$

One of the diagnostic challenge in ACS is in differentiating between Unstable angina and NSTEMI. The Unstable angina and NSTEMI are closely related conditions: their pathophysiologic origins and clinical presentations are similar, but they differ in severity. A diagnosis of NSTEMI can be made when the ischemia is sufficiently severe to cause myocardial damage that results in the release of a biomarker of myocardial necrosis into the circulation ${ }^{(6)}$ Raised cardiac Troponin $\mathrm{T}$ concentrations are now considered the standard biochemical marker for the diagnosis of myocardial Infarction ${ }^{(7)}$. They are even helpful in detecting myocardial damage in a patient who presents for assessment several days after the onset of symptoms.

\section{Methods}

A descriptive study was conducted with data of platelet indices and Troponin $\mathrm{T}$ values in patients admitted with acute coronary syndrome between January-June 2016 in Jayadeva institute of Cardiac Science and Research Institute (SJ ICSRI), MYSORE.

The Jayadeva Institute of Cardiac Science and Research Institute, located in Mysore. It is a 110 bedded teaching institution with 80-100 outpatient visits and about 5-10 inpatient admissions towards every day. Nearly 1-2 cases of Acute coronary syndromes are admitted each day in the intensive care unit. Treating Doctors order a complete blood count and Troponin-T for all patients with ACS as a standard care. Since past eight years, all blood counts are being performed using an electronic cell counter (sysmex) and HSST using an instrument called Roch -Hitachi-Cobas-e-411, in the Laboratory. This electronic cell counter and Roch -Hitachi-Cobas-e-411 meets all the quality control requirements and is periodically standardized

\section{Inclusion Criteria}

All patients with values of blood count and Troponin $\mathrm{T}$ of patients with diagnosis as having Acute Coronary syndrome like Acute Myocardial infarction (STEMI / NSTEMI) or Unstable angina admitted between January-June 2016.

\section{Exclusion Criteria}

Those cases, 1) Where the electronic cell count and Troponin- $\mathrm{T}$ values were not obtained within 12 hours of hospital admission (as anti-platelet drug therapies can modify platelet indices), and 2) Where the blood sample either had clot or a marked anti-coagulant effect (as reported on peripheral smear), were excluded.

\section{Study Procedures}

SJICS\&R hospital has an electronic hospital records system, and records of all diagnosis, electronic cell counts and HSST values are maintained in an electronic form. The clinical details, the electronic cell counts and Troponin $\mathrm{T}$ values of the same patient are linked using a four digit unique identifier, known as a laboratory number.

Hospital information system was used to obtain case sheets of all eligible cases. Personal identifiers in the list such as name or address were masked. The date and time of admission for these cases were obtained from the hospital information system. Then the electronic cell count and Troponin $\mathrm{T}$ data for these eligible patients were obtained using the lab number as a reference. This data is also maintained in an electronic data-base form in the Laboratory. The time interval between hospital admission cell count along with Troponin $\mathrm{T}$, presence of clot or anticoagulation was noted to determine any exclusion. Platelet count and platelet volume indices (MPV and PDW) and Troponin $\mathrm{T}$ were noted in cases. Approval was obtained from the institute's committee to conduct this study. 
Statistical Analysis: Data was analysed using SPSS software.

\section{Results}

Table 1

\begin{tabular}{|l|c|c|}
\hline Frequency & \multicolumn{2}{|c|}{ Percent } \\
\hline 31 to 40 & 11 & 11.0 \\
\hline 41 to 50 & 26 & 26.0 \\
\hline 51 to 60 & 25 & 25.0 \\
\hline 61 to 70 & 24 & 24.0 \\
\hline 71 to 80 & 8 & 8.0 \\
\hline$>80$ & 6 & 6.0 \\
\hline Total & 100 & 100 \\
\hline
\end{tabular}

Among the 100 cases studied majority of cases were in the age group of 41-50yrs.
Figure 1: Age wise distribution of the population

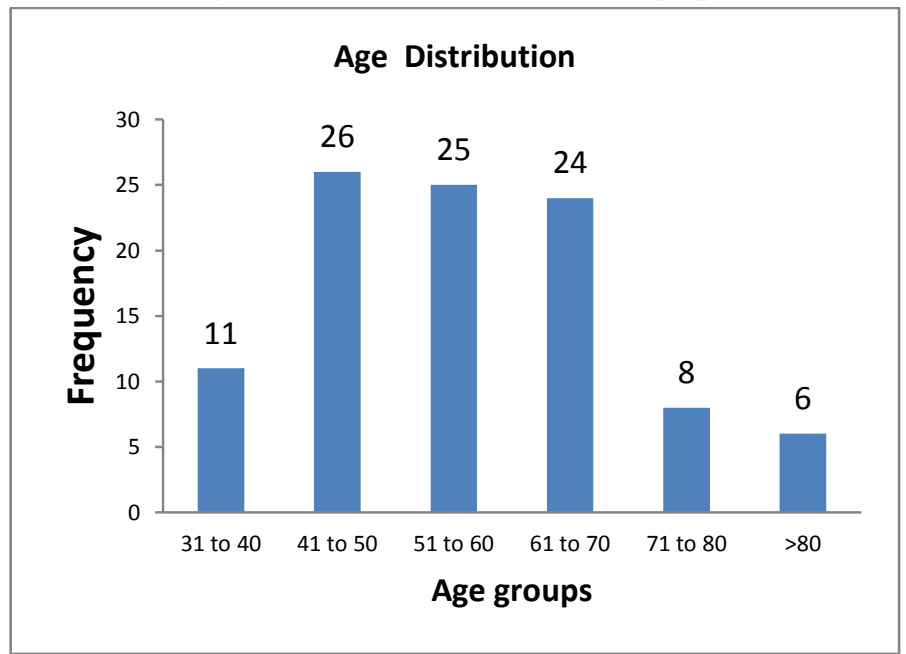

Table 2: Observed values in the study

\begin{tabular}{|l|c|c|c|c|c|}
\hline & No of cases & Min value & Max value & Mean value & S D \\
\hline Platelet count & 100 & 1.0 & 2.4 & 2.5385 & 2.3146 \\
\hline MPV & 100 & 6.30 & 10.10 & 7.76 & 0.74079 \\
\hline PDW & 100 & 10.40 & 16.00 & 12.22 & 1.10267 \\
\hline Pct & 100 & 0.04 & 0.35 & 0.16 & 0.05696 \\
\hline Troponin T & 100 & 0.01 & 9.48 & 0.7521 & 1.5242 \\
\hline Valid N & 100 & \multicolumn{3}{|l}{} \\
\hline
\end{tabular}

The mean values of MPV=7.76 fl, $\mathrm{PDW}=12.2 \%, \mathrm{Pct}=0.16 \%$ and platelet count $=2.53$ lacs. The mean value of Troponin $\mathrm{T}$ was $0.75 \mathrm{ng} / \mathrm{ml}$

Table 3: Gender wise distribution of platelet indices and Troponin $T$

\begin{tabular}{|c|c|c|c|c|c|c|c|c|c|c|c|c|}
\hline \multirow{4}{*}{ Gender } & & \multicolumn{2}{|c|}{ Count } & \multicolumn{2}{|c|}{ MPV } & \multicolumn{2}{|c|}{ PDW } & \multicolumn{2}{|c|}{ PCT } & \multicolumn{2}{|c|}{ Trop t } & \multirow{2}{*}{$\mathrm{N}$} \\
\hline & & Mean & SD & Mean & SD & Mean & SD & Mean & SD & Mean & SD & \\
\hline & Male & 2.12 & 0.61 & 7.77 & 0.75 & 12.25 & 1.12 & 0.15 & 0.04 & 0.8 & 1.73 & 72 \\
\hline & Female & 2.61 & 0.98 & 7.74 & 0.71 & 12.16 & 1.06 & 0.19 & 0.07 & 0.61 & 0.73 & 28 \\
\hline$P$ value & & \multicolumn{2}{|c|}{0.004} & \multicolumn{2}{|c|}{0.87} & \multicolumn{2}{|c|}{0.72} & \multicolumn{2}{|c|}{0.003} & \multicolumn{2}{|c|}{0.57} & \\
\hline
\end{tabular}

The mean values of the parameters were analysed with respect to sex. There was significant difference in mean values of platelet $\operatorname{count}(\mathrm{p}$ value $=0.004)$ and Pct $(\mathrm{p}$ value $=0.003)$ across males and females .

\section{Discussion}

Platelet parameters, which include the plateletcrit (PCT), platelet distribution width (PDW) and mean platelet volume (MPV), have been available in the laboratory routine investigations using blood cell counters for several years. Although many studies have attempted to establish clinical correlations to support their application, these parameters are still not used much owing to methodological problems which make standardization and the determination of reference values difficult. ${ }^{(8)}$ Generalized platelet activation occurs during the acute coronary event, where the increase rate of platelet consumption at the site of atherosclerotic plaque rupture leads to the release of large size platelets from the bone marrow. This activation process results in signalling pathways that induce platelets to change their shape (metamorphosis) and size ${ }^{(5)}$ and become more active in secreting thromboxane A2 and ADP into the circulation. Larger platelets are more adhesive and tend to aggregate more than smaller ones ${ }^{(6)}$. They contain more secretory granules and mitochondria and are known to be more active than smaller platelets ${ }^{(2)}$

In ACS the significance of Cardiac troponin $\mathrm{T}$ is because it is a myofibrillar protein, only expressed in myocardial cells, for which highly specific assays have been developed that allow precise differentiation of cardiac and skeletal muscle 
damage Cardiac troponins $\mathrm{T}$ and $\mathrm{I}$ are the preferred markers for myocardial injury as they have the highest sensitivities and specificities for the diagnosis of acute myocardial infarction Troponin is a protein released from myocytes when irreversible myocardial damage occurs. It is highly specific to cardiac tissue and accurately diagnoses myocardial infarction with a history of ischemic pain or ECG changes reflecting ischemia. Cardiac troponin level is dependent on infarct size, thus providing an indicator for the prognosis following an infarct. Because troponin levels remain elevated for a prolonged period ( 5 to 14 days) after myocardial necrosis, their usefulness in detecting recurrent myocardial damage is limited. However, they are helpful in detecting myocardial damage in a patient who presents for assessment several days after the onset of symptoms ${ }_{(15)}$

In an Indian study conducted by $\mathrm{R}$ Badiger et al ${ }^{(3)}$, Khandekar et al ${ }^{(5)}$, Randheer pal et al ${ }^{(4)}$, the authors suggested that all platelet volume indices are significantly raised in patients with acute myocardial infarction and unstable angina patients as compared to those with stable coronary artery disease. Also studies on Troponin $\mathrm{T}$ by $\mathrm{S}$ Sharma et al ${ }^{(10)}$, Amit et al ${ }^{(15)}$ and $\mathrm{Ru}-\mathrm{Yi} \mathrm{Xu}$ et al ${ }^{(19)}$ showed that rise in Troponin $\mathrm{T}$ Values above $0.01 \mathrm{ng} / \mathrm{ml}$ is a risk factor for Acute coronary syndrome.

In contrast to the results obtained in these studies, we did not find any significant difference between platelet count and volume indices of cases. Our findings are in concordance with the findings of A Bhayana et al ${ }^{(1)}$ and Damodar et al ${ }^{(9)}$ who also concluded that raised MPV is not a risk factor for development of acute coronary syndrome. But the mean value of Troponin $\mathrm{T}$ with respect to gender was $0.8 \mathrm{ng} / \mathrm{ml}$ in males and $0.61 \mathrm{ng} / \mathrm{ml}$ in females. Hence Troponin $\mathrm{T}$ a risk factor in $\mathrm{A}$ cute Coronary Syndrome.

\section{Conclusion}

Mean Platelet Volume was not found to be a risk for Acute Coronary syndrome in our study. More research on this subject should be carried out on larger number of subjects before considering mean platelet volume as a risk factor and a marker of progression of disease for ACS. But Troponin T can be considered as a prognostic marker in patients with acute coronary syndrome.

\section{References}

1. A Bhayana, D Joshi. Is Large Platele Size A Risk Factor For Acute Coronary Syndrome : A Retrospective CaseControlStudy.JMGIS,September2009,vol1 4,no(ii),52-55

2. Abdul S Assiri, Abdul-Moneim Jamil, et all. Diagnostic importance of platelet parameters in patients with acute coronary syndrome admitted to a tertiary care hospital in southwest region ,Saudi Arabia. J Saudi Heart Association 201;24:17-21

3. Dr. R Badiger, Dr. A Hosalli et al, Platelet indices in patients of acute coronary syndrome patients in tertiary care hospital. International Journal of Applied Research 2015; 1(13): 731-734

4. Daniel Agustín Chirino Navarta, Ariel Monteros, GracielaTrejo, et al.Mean Platelet Volume as Prognostic Marker in Patients with Acute Coronary Syndrome. ARGENTINE JOURNAL OF CARDIOLOGY / VOL $83 \mathrm{~N}^{\circ} 4$ / AUGUST 2015

5. E Vagdatli, E Gounari, E Lazaridou,et al. Platelet distribution width: a simple, practical and specific marker of activation of coagulation. Hippokratia. 2010 JanMar; 14(1): 28-32

6. S Maynard, I Menown, and A Adgey. Troponin $\mathrm{T}$ or troponin $\mathrm{I}$ as cardiac markers in ischaemic heart disease. Heart. 2000 Apr; 83(4): 371-373

7. S Sharma, $P$ G Jackson, and J Makan. Cardiac troponins. J Clin Pathol. 2004 Oct; 57(10): 1025-1026.

8. Trip MD, Cats VK, van Capelle FJL, et al. Platelet hyperreactivity and prognosis in survivors of myocardial infarction. New Engl J Med 1990;322:1549-54. 
9. Damodar S, Ganesh KV,Murthy S. Mean Platelet Volume does not predict risk of myocardial infarction or coronary artery disease in Indian patients. Platelets 2008;19(1):80-8.

10. RandheerPal, Rajeev Bagarhatta, et al. Mean Platelet Volume in Patients with Acute Coronary Syndromes: A Supportive Diagnostic Predictor. JCDR 2014Aug, Vol8(8):MC01-MC04

11. Khandekar MM, Khurana AS, Deshmukh SD, Kakrani AL, Katdare AD, Inamdar AK. Platelet volume indices in patients with coronary artery disease and acute myocardial infarction: an Indian scenario. J Clin Pathol 2006;59:146-149

12. Thayse Vanessa Giovanetti, Aguinaldo José do Nascimento, and Josiane Padilha de Paula. Platelet indices: laboratory and clinical application. Rev Bras Hematol Hemoter. 2011; 33(2): 164-165.

13. Professor Dr. Riaz Ahmad BhuttaDr. Naheed Afroz Syed,Dr. Asad Ahmad, M.D. Platelet Indices .Labpedia.net

14. Amar R. Shah1, Sanjay N. Chaudhari2, Role Of Platelet Parametres In Diagnosing Various Clinical Condition.NJMR 2013;3(2):162-165.

15. Amit Kumar, MD and Christopher P. Cannon, MD. Acute Coronary Syndromes: Diagnosis and Management, Part I. Mayo Clin Proc. 2009 Oct; 84(10): 917-938.

16. Karpatkin S. Heterogeneity of human platelets. II.Functional evidence suggestive of young and oldplatelets. J Clin Invest 1969; 48:1083-1087.

17. Lakkis N, Dokainish H, Abuzahra M, Tsybouley V,Jorgensen J, Ponce De Leon A, Saleem A.Reticulated Platelets in Acute Coronary Syndrome:A Marker of Platelet Activity. JACC 2004;44(10):2087-98.

18. Cameron EA, Phillips R, Ibbatson RM, et al.Platelet size in myocardial infarction. BMJ1983;287:449-51.
19. Ru-Yi Xu,1 Xiao-Fa Zhu,1 Ye Yang.Highsensitive cardiac troponin T. J Geriatr Cardiol. 2013 Mar; 10(1): 102-109.

20. Silvia Cristina Costa, Carmen Guilherme de Matos Vinagre, Ana Paula Marte , Platelet Indices in Patients with Acute Coronary Syndrome.JBM 2015;3:71-76.

21. Corash L, Tan H, Grolnick HR. Heterogeneity of human whole blood platelet subpopulations. I.Relationship between buoyant density, cell volume and ultrastructure. Blood 1977;49:71-87.

22. Thompson CB, Eaton KA, Princiotta SM, et al. Size-dependent platelet subpopulation: relationship of platelet volume to ultrastructure, enzymaticactivity and function. Br J Haematol 1982;50:509-20. 